\title{
Glass Transition and Aging in Dense Suspensions of Thermosensitive Microgel Particles
}

\author{
Eko H. Purnomo, ${ }^{*}$ Dirk van den Ende, ${ }^{\dagger}$ Siva A. Vanapalli, ${ }^{\ddagger}$ and Frieder Mugele \\ Physics of Complex Fluids, IMPACT, Department of Science and Technology, University of Twente, \\ P.O. Box 217, 7500 AE Enschede, The Netherlands
}

(Received 1 July 2008; published 2 December 2008)

\begin{abstract}
We report a thermosensitive microgel suspension that can be tuned reversibly between the glass state at low temperature and the liquid state at high temperature. Unlike hard spheres, we find that the glass transition for these suspensions is governed by both the volume fraction and the softness of the particles, where softer suspensions form a glass at higher effective volume fractions. In the glass state, these suspensions show aging where the relaxation times increase linearly with age, irrespective of the degree of particle softness. This relaxation scaling is in contrast with hard sphere behavior but consistent with the soft glassy rheology model.
\end{abstract}

DOI: 10.1103/PhysRevLett.101.238301

PACS numbers: $83.80 . \mathrm{Hj}, 83.60 . \mathrm{Bc}, 83.80 . \mathrm{Kn}$

Soft glassy materials (SGMs) exhibit distinct rheological behavior. Under small stresses, they behave like a solid $\left(G^{\prime}>G^{\prime \prime}\right)$ on experimental time scales. However, at very long time scales, they flow. Such rheological features characteristic of soft glassy behavior have been found in many materials including colloidal suspensions [1-8], emulsions [9], foams [10], and living cells [11]. The microscopic dynamics of these soft materials reveals signatures of glassy behavior including metastability, dynamic heterogeneities, intermittency, and kinetic arrest [12]. Upon increasing the mass concentration of a suspension of colloidal hard spheres, the system undergoes a glass transition at a volume fraction of $\phi_{g} \simeq 0.58$. In contrast, for soft spheres [13] or star polymers [14], the glass transition can be induced by varying not only the mass concentration but also the particle size (via $p \mathrm{H}$ or temperature). Because soft particles can easily be deformed, they flow under an infinitesimal shear stress even at volume fractions higher than 0.58, raising the question: How does the volume fraction at the glass transition depend on the softness or elasticity of the particles?

Another characteristic signature of soft glassy materials is that they often show aging where rheological properties continuously evolve with time. Its universality has been demonstrated $[1,6,7]$ by scaling the evolution of the dynamic properties on the age of the concentrated suspension. Aging has also been explored in colloidal hard sphere suspensions by measuring the mean squared displacement of the particles as a function of time, at different ages of the sample, using video microscopy [2,3]. These measurements showed that the mean relaxation time increases for hard spheres sublinearly with the age of the suspension. In contrast, using rheological measurements we recently discovered that soft microgel particle suspensions $[4,5]$ in the glassy state show a frequency dependence of the viscoelastic moduli that scales linearly with the age of the suspension, implying that the mean relaxation time also scales linearly with sample age. This difference in the aging behavior between hard and soft sphere systems raises the second question: How does the particle softness affect aging behavior?

In this Letter, we address both issues using thermosensitive colloidal suspensions. These suspensions form a unique class of SGMs that is well suited for studying mechanical behavior in the vicinity of glass transition because both the volume fraction and the particle softness can be tuned independently by varying the particle size (via the temperature $[4,5,13])$ and the number density of particles (via the mass concentration) in a controlled way.

We apply the soft glassy rheology (SGR) model to interpret the observed aging behavior in the glassy state as well as to characterize the liquid-glass transition. In this model $[15,16]$ the steric hindrance against relaxation of stresses inside the particles, characterized by an elasticity $G_{p}$, is represented by a complex landscape of energy wells with an average depth $X_{g}$. Stress relaxation is conceived as hopping of a particle from one well to another. As in activated rate processes, the hopping probability depends on the depth of the well as well as the effective noise temperature $X$. We demonstrate that the degree of glassiness (quantified by the relative noise temperature $x=$ $X / X_{g}$ ) of a thermosensitive microgel suspension can be tuned continuously and reversibly by varying the thermodynamic temperature $T$. The aging behavior of the viscoelastic moduli observed in the glassy state, where $x<1$, is not observed above the glass transition, where $x>1$. Both below and above the glass transition, the moduli are quantitatively described by the SGR model.

The thermosensitive colloidal suspensions we use in this study contain core-shell microgel particles. These particles have a poly-N-isopropyl acrylamide (polyNipam) core and a poly-N-isopropylmethacrylamide shell [17]. The size of these particles depends more gradually on the temperature than for pure polyNipam particles, resulting in a broader temperature range to tune the volume fraction $\phi$ of the suspension. The dependence $\phi(T, c)$ was determined by measuring the relative Einstein viscosity $\eta_{r}(c)=$ $1+5 / 2 \phi$ at $T_{0}=24{ }^{\circ} \mathrm{C}$ for relative mass concentrations 
$c$ below $0.005 \%$ weight by weight $(\mathrm{w} / \mathrm{w})$, resulting in $\phi\left(T_{0}, c\right)=\kappa c$, with $\kappa=42 \pm 1$. For other temperatures the volume fraction is $\phi(T, c)=\phi\left(T_{0}, c\right)\left[R(T) / R\left(T_{0}\right)\right]^{3}$, where the particle radius $R(T)$ was obtained from static light scattering experiments. Experiments were performed at volume fractions, as defined in this way, between 0.6 and 3.0. Note that the same volume fraction can be obtained for different concentrations by adjusting the temperature. However, at constant volume fraction the particle softness decreases with increasing concentration, due to an increasing intraparticle polymer concentration.

All experiments were carried out using a Haake RS600 rheometer with a cone and plate geometry (diameter $60 \mathrm{~mm}$, angle $2^{\circ}$ ). A vapor lock was used to avoid evaporation, and the temperature of the shielding was kept approximately $5^{\circ} \mathrm{C}$ above the plate temperature to prevent condensation. The rheometer was loaded at $44{ }^{\circ} \mathrm{C}$ and then cooled down to the experimental temperature. Prior to any measurement, the suspension was rejuvenated by applying, during $60 \mathrm{~s}$, a stress $\sigma_{q}$ well above (typically twice) the yield stress $\sigma_{y}$. Next, the elastic modulus $G^{\prime}$ and the loss modulus $G^{\prime \prime}$ were measured as a function of the frequency $\omega$, for several waiting times $t_{w}$. The aging time $t$ is defined as the total time lap from the end of the rejuvenation to the moment of data acquisition $[4,5]$.

Figure 1(a) shows the frequency dependence of the moduli of a $7 \% \mathrm{w} / \mathrm{w}$ suspension at $25^{\circ} \mathrm{C}$. The aging is clearly demonstrated by the $G^{\prime \prime}$ behavior: At a given frequency below $1 \mathrm{rad} / \mathrm{s}$, it strongly decreases with increasing time. In Fig. 1(b), the same moduli are plotted versus $\omega t$ instead of $\omega$. Now they collapse onto a master curve for $\omega t<300$, which implies that the viscoelastic relaxation times scale with time $t$. For $\omega t>300$, where the moduli are dominated by local viscous and Brownian effects, they do not collapse, as discussed in more detail in [4]. This aging behavior of the moduli can be described using the energy landscape picture of the SGR model [15]. As particles escape more readily from shallow wells, the deeper ones become more populated as the system gets older. Simultaneously, the average escape time, i.e., the structural relaxation time, increases as well. The slight increase of $G^{\prime}$ and the decrease of $G^{\prime \prime}$ with increasing $\omega t$ (for $\omega t<300$ )
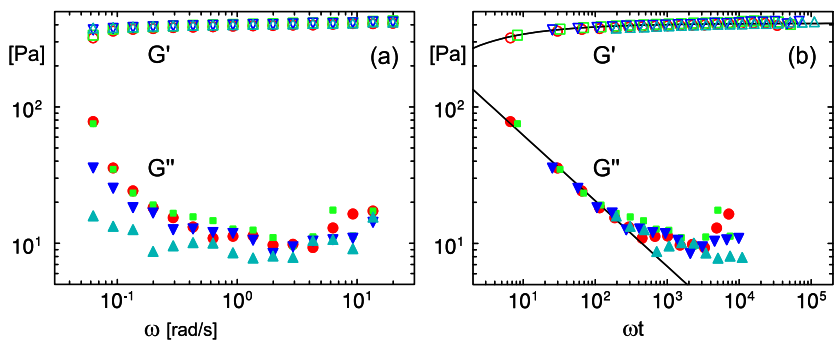

FIG. 1 (color online). $\quad G^{\prime}$ (open symbols) and $G^{\prime \prime}$ (solid symbols) of a $7 \% \mathrm{w} / \mathrm{w}$ suspension at $25^{\circ} \mathrm{C}$ plotted versus $\omega$ (a) or $\omega t$ (b) for $t_{w}=3(\bigcirc), 30(\square), 300(\nabla)$, and $3000 \mathrm{~s}(\triangle)$. Lines represent the SGR model $\left(x=0.55, G_{p}=410 \mathrm{~Pa}\right)$. in Fig. 1(b) can be explained in terms of stress yielding of the particles. This yielding dissipates energy and lowers the number of strained particles. As $\omega$ increases, the time $1 / \omega$ available for yielding during one cycle decreases, resulting in a higher $G^{\prime}$ and lower $G^{\prime \prime}$. The same reasoning explains the decrease in $G^{\prime \prime}$ and the slight increase in $G^{\prime}$ upon aging, since the particles occupy increasingly deeper traps implying a decreasing yielding rate. The relative noise temperature $x$ was obtained (together with the particle elasticity $G_{p}$ ) by fitting the SGR model [Eq. (43) in [16]] to the experimental data. The value of $x=0.55 \pm$ 0.02 , which was obtained at $T=25^{\circ} \mathrm{C}$, indicates that the suspension is deep in the glassy state.

To show the tunability of the glassiness of these suspensions, we measured the viscoelastic moduli at various temperatures and waiting times. The symbols in Fig. 2 show the frequency dependence of the moduli for various temperatures close to the glass transition $T_{g}$ of a sample with $c=7 \%$. The data for other mass concentrations look qualitatively similar. In Figs. 2(a) and 2(b), the moduli, measured at 35 and $37^{\circ} \mathrm{C}$, respectively, have been plotted versus $\omega t$ for several aging times $t$. The collapse of the data again implies that the system ages. Because of the swelling of the particles, the suspension is in a glassy state and behaves solidlike, i.e., $G^{\prime}>G^{\prime \prime}$. In Figs. 2(c) and 2(d), the moduli, measured at various ages for 38 and $40^{\circ} \mathrm{C}$, respectively, have been plotted versus $\omega$. Now the suspension behaves liquidlike, i.e., $G^{\prime}<G^{\prime \prime}$, and the moduli are independent of the aging time. At $38^{\circ} \mathrm{C}, G^{\prime}$ increases faster than $G^{\prime \prime}: G^{\prime} \sim \omega^{1.2}$ and $G^{\prime \prime} \sim \omega$. At $40^{\circ} \mathrm{C}$, the suspension shows at low frequencies a Maxwellian behavior: $G^{\prime} \sim \omega^{2}$ and $G^{\prime \prime} \sim \omega$. Both in the glass and in the liquid state, the SGR model (curves in Fig. 2) describes the measured data
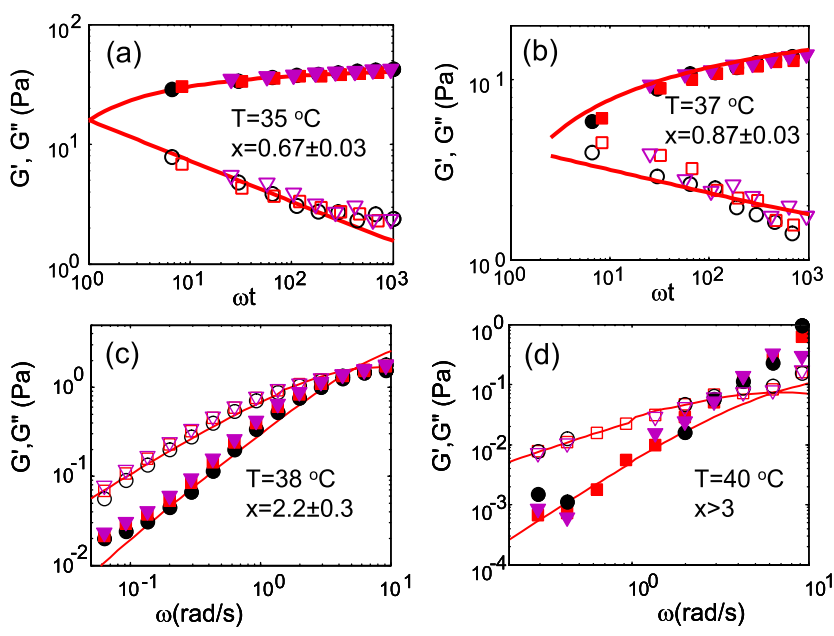

FIG. 2 (color online). Evolution of $G^{\prime}$ (solid symbols) and $G^{\prime \prime}$ (open symbols) of the $7 \% \mathrm{w} / \mathrm{w}$ suspension from glassy, at $T=$ 35 (a) and $37^{\circ} \mathrm{C}$ (b), to liquid behavior at $T=38$ (c) and $40^{\circ} \mathrm{C}$ (d). Lines are the best fitting SGR curves. Data in (a) and (b) were plotted versus $\omega t$ to collapse the curves for $t_{w}=3(\bigcirc), 30$ $(\square)$, and $300 \mathrm{~s}(\nabla)$. Data in (c) and (d) were plotted versus $\omega$ to reach collapse. 
quite well. The glass transition occurs (for this specific concentration) between 37 and $38^{\circ} \mathrm{C}$ and can be tuned reversibly without any noticeable hysteresis.

Several criteria can be used to identify the glass transition in rheological data, such as the ratio $G^{\prime} / G^{\prime \prime}$ at a fixed frequency or the presence or absence of aging. In the following, we chose to use the relative noise temperature $x$ as defined in the SGR model $[15,16]$ to identify the transition. We stress, however, that the conclusions which we will draw below hold independent of this specific choice.

To obtain the relative noise temperature $x$ as a function of $T$ and $c$, the measurements were extended to several mass concentrations. Again $x$ and the particle elasticity $G_{p}$ were obtained from a fit of the SGR model to the experimental data. The results are shown in Fig. 3(a). The behavior of $x(T, c)$ reflects the transition from the glassy state $(x<1)$ at low $T$ to the liquid state $(x>1)$ at high $T$. The transition temperature $T_{g}=T(x=1)$ is found to increase with increasing mass concentration. This is expected since, at constant temperature, the volume fraction is higher for higher mass concentrations. Hence, the temperature at which the particles have enough space to flow freely will also be higher. Extrapolating from the well-known critical volume fraction of hard spheres, one might expect that the data should collapse if we replot them as a function of the (effective) volume fraction. However, this is not the case. In contrast, we observe from Fig. 3(b) that the critical volume fraction increases from 0.85 at the highest mass concentration to 1.4 at the lowest.

From the data in Fig. 3(a), the transition temperature $T_{g}$ at a given mass concentration $c$ can be obtained by determining the temperature for which $x(T, c)=1$. Figure 3(c) shows that, within the concentration range considered, $T_{g}$
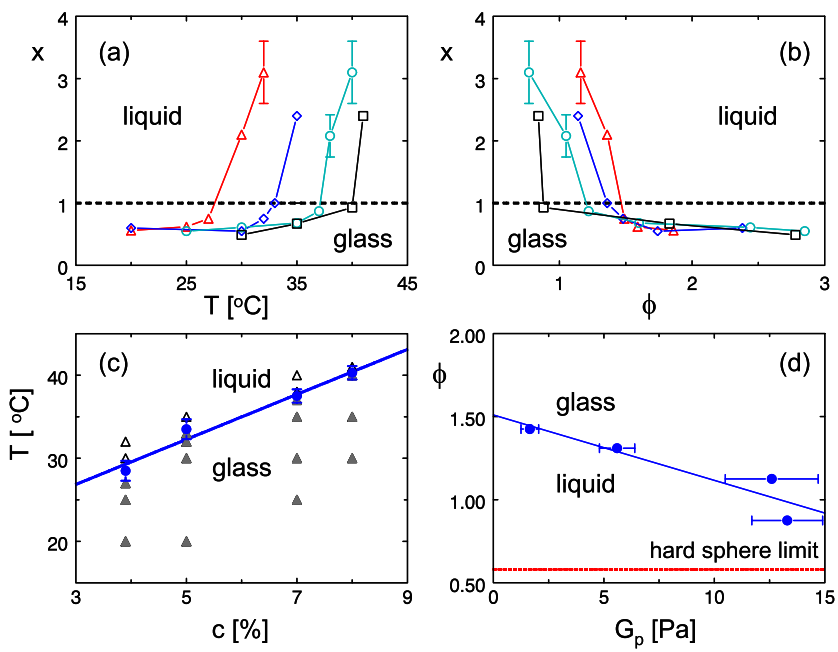

FIG. 3 (color online). (a) Relative noise temperature $x$ versus $T$ at $c=3.9(\triangle), 5(\diamond), 7(\bigcirc)$, and $8 \% \mathrm{w} / \mathrm{w}(\square)$. (b) The same data as a function of $\phi$. (c) Phase behavior ( $\triangle$ : liquid, $\mathbf{\Delta}$ : glass) in the $c$-T plane and the transition temperature $T_{g}(c)$ (line). (d) Transition volume fraction $\phi_{g}$ versus the particle elasticity $G_{p}$. Lines are a guide to the eye. increases almost linearly with the mass concentration. To elucidate the dependence of the glass transition on the volume fraction and the particle softness, the volume fraction at the transition $\phi_{g}$ [obtained from Fig. 3(b)] is plotted in Fig. 3(d) versus the particle elasticity at the transition: $G_{p}\left(T_{g}(c), c\right)$. The values for $G_{p}(T, c)$ were obtained from the viscoelastic data, as described above. Indeed, the volume fraction at the transition decreases with increasing elasticity and tends to the hard sphere limit $\left(\phi_{g} \sim 0.58\right)$ for high elasticity.

As mentioned before, we can determine the transition temperature as a function of the mass concentration [Fig. 3(c)] without invoking SGR, using the $\omega t$ or $\omega$ dependence of the moduli measured at different aging times, as criterion. All data points in Fig. 3(a) above the line $x=1$ correspond to age-independent moduli. At those concentrations and temperatures, the suspensions are in the liquid state [ $\triangle$ in Fig. 3(c)]. All points below this line correspond to age-dependent moduli. At these concentrations and temperatures, the suspensions are in the glass state [ $\mathbf{\Delta}$ in Fig. 3(c)]. The transition temperature at a given concentration is now estimated from this phase diagram. Using the high frequency limit $G_{\infty}^{\prime}$ as a measure of the particle elasticity $G_{p}$, the same model independence can also be established for the data in Fig. 3(d). Hence our conclusions are independent of the SGR model. Moreover, these results show that the glass transition, as marked by the switch from the $\omega t$ dependence of the moduli to the $\omega$ dependence, indeed takes place at $x=1$, as predicted within the SGR model.

The dependence of $T_{g}$ on the mass concentration can be explained in terms of the particle softness. When we consider a microgel particle in the suspension, the particle collapses as we increase the temperature. The collapse consequently increases the polymer density inside the particle, and therefore it behaves harder (i.e., has a higher elasticity) than a fully swollen particle. From Fig. 3(a), one observes that for a suspension with a higher mass concentration (i.e., a higher particle number density) the transition occurs at a higher temperature, which indicates that the individual particles are less swollen and harder compared
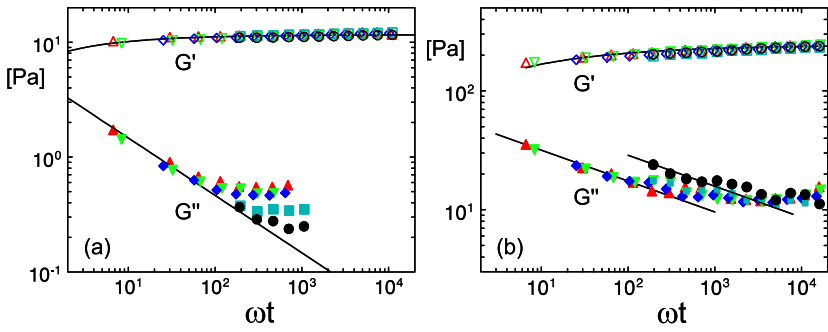

FIG. 4 (color online). $\quad G^{\prime}$ (open symbols) and $G^{\prime \prime}$ (solid symbols) of two different suspensions with $\phi=1.85$ plotted versus $\omega t$ for $t_{w}=3(\triangle), 30(\nabla), 300(\diamond), 3000(\square)$, and $30000 \mathrm{~s}(\bigcirc)$. (a) $c=3.9 \% \mathrm{w} / \mathrm{w}$ at $20{ }^{\circ} \mathrm{C}$; lines are the best fitting SGR curves for $\omega t<100\left(x=0.53 \pm 0.02, G_{p}=12 \pm 1 \mathrm{~Pa}\right)$. (b) $c=8 \% \mathrm{w} / \mathrm{w}$ at $35^{\circ} \mathrm{C}\left(x=0.72 \pm 0.03, G_{p}=245 \pm 30 \mathrm{~Pa}\right)$. 
to those at a lower mass concentration at the same volume fraction. Because both the particle volume fraction and its elasticity affect the glass transition of the suspension, plotting the relative noise temperature $x(\phi, c)$ as function of their volume fraction $\phi$ [see Fig. 3(b)] does not result in a single curve.

The effect of softness is also illustrated in Fig. 4, where the moduli of two different suspensions with the same volume fraction are shown. Despite the same volume fraction, the elastic modulus of the $8 \%$ suspension is about $20 \times$ larger than the one of the $3.9 \%$ suspension. Fitting the SGR model to the data, we obtain $G_{p}=245 \pm 30$ and $12 \pm 1 \mathrm{~Pa}$ and $x=0.53$ and 0.72 , respectively. This indicates that both suspensions are deep in the glassy state, but the particles in a 3.9\% suspension are softer than in an $8 \%$ suspension at the same volume fraction, in line with the discussion of Fig. 3. Moreover, we observe that the aging of the $3.9 \%$ suspension continues at least to $t_{w}=3 \times 10^{4} \mathrm{~s}$, whereas the $8 \%$ suspension ages only up to $t_{w} \simeq 6 \times 10^{3} \mathrm{~s}$, as concluded from the shift with a factor of 5 of the curve at $3 \times 10^{4} \mathrm{~s}$ with respect to the other curves in Fig. 4(b). This termination of aging for harder particles can be rationalized as a total arrest of the dynamics of a particle by its neighboring particles. Harder particles are less deformable; therefore, the length scale of the rearrangements diverges. Although this is in line with observations by Crassous et al. [18], it cannot be explained within the SGR model.

The incomplete arrest in the glassy state due to the softness of the particle interactions also explains the creep behavior below the yield stress and the flow behavior above it $[6,8]$. After a fast stress pulse below the yield value $\sigma_{y}$, these systems respond elastically without any irreversible deformation. However, when the same stress pulse is applied much more slowly, the system does show irreversible deformation, which is larger for shorter ages. This can be attributed to yielding events between neighboring particles, which are less probable for older systems. Applying a stress above $\sigma_{y}$, the suspension flows and no aging is observed, because the yielding of the particles is now determined by the shear.

In conclusion, we demonstrated that the glass transition is governed by the volume fraction and the softness of the particles: Suspensions of softer particles require a higher volume fraction to become glassy. We determined $T_{g}(c)$, below which the system is in the glassy state where it shows aging; i.e., the mechanical relaxation times scale linearly with the age of the suspension. Above $T_{g}(c)$, the system behaves liquidlike and no aging is observed. The SGR model describes the time and frequency dependence of the moduli above and below $T_{g}$ quantitatively correctly. The volume fraction at the liquid-glass transition tends to the hard sphere limit $\left(\phi_{g} \simeq 0.58\right)$ as the particles get harder.

We thank W. Richtering and M. Keerl for sample preparation, M. Ballauff, M. Fuchs, S. Nagel, and P. Sollich for fruitful discussions, and J. Mellema for initiating the project. This work has been supported by the Foundation for Fundamental Research on Matter (FOM), which is financially supported by the Netherlands Organization for Scientific Research (NWO).

*Present address: Department of Food Science and Technology, Bogor Agricultural University, Indonesia.

Corresponding author.

h.t.m.vandenEnde@utwente.nl

*Present address: Department of Chemical Engineering, Texas Tech University, Lubbock, TX 79409, USA.

[1] Michel Cloitre, Régis Borrega, and Ludwik Leibler, Phys. Rev. Lett. 85, 4819 (2000).

[2] R. E. Courtland and E. R. Weeks, J. Phys. Condens. Matter 15, S359 (2003).

[3] C. Derec, A. Ajdari, G. Ducouret, and F. Lequeux, C. R. Acad. Sci. Ser. IV 1, 1115 (2000); C. Derec, G. Ducouret, A. Ajdari, and F. Lequeux, Phys. Rev. E 67, 061403 (2003); V. Viasnoff and F. Lequeux, Phys. Rev. Lett. 89, 065701 (2002); D. Bonn, P. Coussot, H. T. Huynh, F. Bertrand and G. Debrégeas, Europhys. Lett. 59, 786 (2002).

[4] E. H. Purnomo, D. vanden Ende, J. Mellema, and F. Mugele, Europhys. Lett. 76, 74 (2006).

[5] E. H. Purnomo, D. van den Ende, J. Mellema, and F. Mugele, Phys. Rev. E 76, 021404 (2007).

[6] Y. M. Joshi and G. R. K. Reddy, Phys. Rev. E 77, 021501 (2008).

[7] G. Ovarlez and P. Coussot, Phys. Rev. E 76, 011406 (2007).

[8] P. Coussot, H. Tabuteau, X. Chateau, L. Tocquer, and G. Ovarlez, J. Rheol. (N.Y.) 50, 975 (2006).

[9] L. Bécu, S. Manneville, and A. Colin, Phys. Rev. Lett. 96, 138302 (2006).

[10] S. Cohen-Addad, H. Hoballah, and R. Höhler, Phys. Rev. E 57, 6897 (1998).

[11] P. Bursac, G. Lenormand, B. Fabry, M. Oliver, D. A. Weitz, V. Viasnoff, J. P. Butler, and J. J. Fredberg, Nature Mater. 4, 557 (2005).

[12] L. Cipelletti and L. Ramos, J. Phys. Condens. Matter 17, R253 (2005).

[13] J. J. Crassous, M. Siebenbürger, M. Ballauff, M. Drechsler, O. Henrich, and M. Fuchs, J. Chem. Phys. 125, 204906 (2006).

[14] M. Kapnistos, D. Vlassopoulos, G. Fytas, K. Mortensen, G. Fleischer, and J. Roovers, Phys. Rev. Lett. 85, 4072 (2000).

[15] P. Sollich, F. Lequeux, P. Hébraud, and M. E. Cates, Phys. Rev. Lett. 78, 2020 (1997); P. Sollich, Phys. Rev. E 58, 738 (1998).

[16] S. M. Fielding, P. Sollich, and M. E. Cates, J. Rheol. (N.Y.) 44, 323 (2000).

[17] I. Berndt, J. S. Pedersen, P. Lindner, and W. Richtering, Langmuir 22, 459 (2006); I. Berndt, J. S. Pedersen, and W. Richtering, J. Am. Chem. Soc. 127, 9372 (2005); I. Berndt and W. Richtering, Macromolecules 36, 8780 (2003).

[18] J. J. Crassous, M. Siebenbürger, M. Ballauff, M. Drechsler, D. Hajnal, O. Henrich, and M. Fuchs, J. Chem. Phys. 128, 204902 (2008). 